\section{Race to explain procrastination}

\author{
Jeffrey D. Schall
}

"WITH the speed of thought" is intended to convey the ultimate in speed. But when, in 1850 , Helmholtz $z^{1}$ clocked the velocity of signal conduction in the nervous system at a modest 50 metres per second, he recognized that mental processes could not be faster than brain processes. This discovery led to the birth of experimental psychology and the development of a new experimental paradigm - the idea was to measure the time taken by subjects to react to different stimuli under different instructions. How brain activity gives rise to behaviour could be understood by relating behavioural reaction time to the time taken by neural pathways to respond to the stimulus and generate the response $\mathrm{e}^{2,3}$. On page 59 of this issue ${ }^{4}$, Carpenter and Williams present evidence for a computational signal that may be a link between neural activity and behavioural reaction time.

When a light flashes in one's peripheral vision, the gaze shifts to look at it with a rapid eye movement called a saccade; the figure shows the brain structures responsible for generating visually guided saccadic eye movements. The neural response to a visual stimulus is conveyed from the retina to central structures such as the superior colliculus and primary visual cortex within 40-100 milliseconds. Visual signals are relayed to other cortical areas and subcortical structures that are ultimately responsible for generating the command to move the eyes. Brain structures such as the superior colliculus and the frontal eye field that are responsible for generating saccades do so with a latency of about $30-50$ ms. Thus, if the interval from the visual stimulus to an eye movement is due solely to conduction along the neural pathways between the retina and the extraocular muscles, then saccades should have latencies ranging from no less than $70 \mathrm{~ms}$ to no more than $150 \mathrm{~ms}$. In fact, though, as Carpenter has pointed out, saccade latencies are typically longer and much more variable than this because the brain seems to procrastinate, probably because of the shift gaze ${ }^{5}$.

Just how the apparent procrastination arises from the nervous system is a mystery. The duration of processing in the brain, we now know, cannot be calculated by a simple addition of the time of axonal conduction and synaptic transmission between successive neurons. This is because no neuron single-handedly influences or is influenced by another neuron. Computations in the cerebral cortex are performed as each neuron integrates over time the weak and vacillating influences of hundreds of other neurons. The outcome of neural processing may be analogous to the coalition of votes among constituents racing to influence a decision.

Carpenter and Williams ${ }^{4}$ employ the concept of a race in the design of a simple stochastic model to account for both the necessity to decide where and when to

\title{
Atom in a cage
}

Nor abstract art, but concrete evidence of something that fullerene specialists had hoped and suspected to be the case, the figure shown here demonstrates that a metal atom can indeed be trapped within a cage of carbon. The contours map the electron density across a section through the metallofullerene $Y @ C_{82}$, the shorthand notation for yttrium in an 82-carbon cage. The pink and swollen bulge inside the circle's lower rim shows the high electron density expected to be associated with the yttrium atom - here clearly ensconced within the blue carbon framework. Theory and experiment had both suggested that this was so; but the new

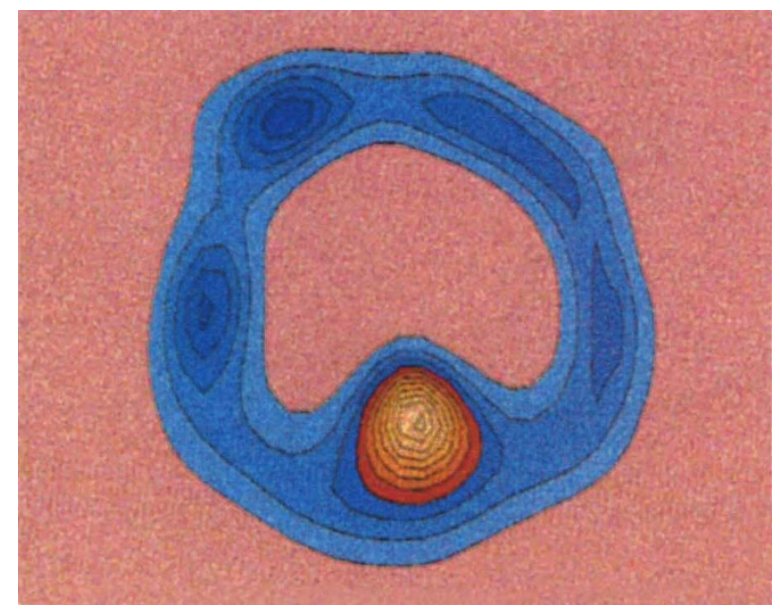

structure, derived from X-ray powder diffraction experiments, may finally allay any doubts. Details of the experiment, carried out by Japanese researchers $M$. Takata et al. at Mie and Nagoya Universities, can be found on page $\mathbf{4 6}$ of this issue.

L. N.

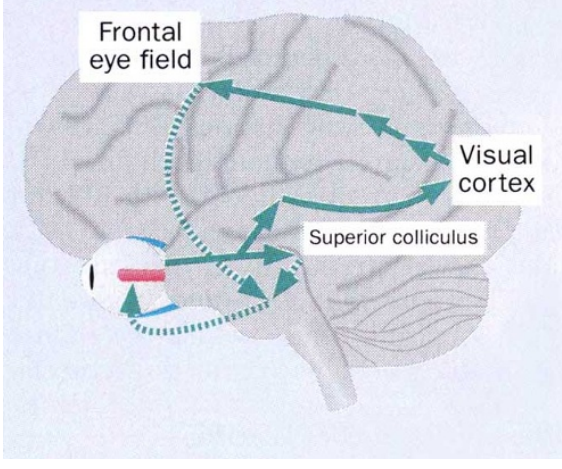

Simplified diagram of the brain circuitry responsible for generating gaze shifts. Solid arrows indicate the flow of visual signals into the brain. Dashed arrows indicate the flow of motor commands out of the brain. Saccadic gaze shifts can be generated by either the superior colliculus or the frontal eye field. The superior colliculus receives visual signals directly from the eye and sends a motor command to circuitry in the brainstem responsible for generating the saccade. In the cortical circuit, visual signals are relayed to visual cortex in the occipital lobe, whereupon multiple streams of processing lead to frontal cortex where the frontal eye field generates commands to move the eyes.

random and systematic variation in the latency to move the eyes when subjects are asked to shift their gaze to a spot of light that jumps to either the left or the right. Unlike typical neural networks with ad hoc elements and mysterious hidden units, this model is a mathematical formulation of a process underlying behavioural performance. And unlike many models of reaction time that seek to explain only central tendencies, this model is designed to account for the entire range and shape of the latency distribution. The model posits the existence of a signal that grows as information about the presence and location of a visual target accumulates. When this signal reaches a threshold, then a movement is triggered.

This general architecture is similar to other reaction time models ${ }^{6}$. In the model of Carpenter and Williams, the random variation of saccade latencies arises from stochastic variability of the rate of growth of the accumulator signal. These authors test the hypothesis that the accumulating signal may correspond to a likelihood ratio of the sort developed in theories about statistical decision processes. Theoretically, the likelihood ratio is an optimum measure on which to base decisions. To investigate whether likelihood ratio is a valid construct, they manipulated the decision process by systematically biasing the presentation of the targets, thereby demonstrating that when the probability of target presentation is changed, the latencies of subjects to shift their gaze to the visual targets varies as predicted by their model.

Clearly, further work should evaluate whether alternative models can simulate 
these results. The model of Carpenter and Williams establishes a benchmark against which to judge competing hypotheses. What is more important, such models are necessary for us to understand how brain processes give rise to behaviour and mental processes. It is commonly said that the brain 'processes' information, but really, instead of directly monitoring information flow in the brain, recordings from neurons only measure changes in discharge rates. Neurophysiologists struggle with the question of what signal a neuron is carrying and therefore what its activity means.

Answers to this fundamental question can come about only through theories of the function of the neural circuit under investigation and its relation to behaviour. Such theories should make use of well formulated signals to perform the desired computations and also specify how those signals are conveyed in neural discharge patterns. For example, models of the oculomotor system use a concept known as 'motor error'. Models of sensory systems are based on the concept that neural activity encodes or represents attributes of sensory stimuli.

Several laboratories have started to investigate the activity of neurons thought

to be involved in perceptual and motor decisions ${ }^{7-4}$. An important implication of Carpenter and Williams' work is that likelihood ratio may serve as an effective measure of how much, over time, the activity of a neuron contributes to a decision. Linking hypotheses like this are needed to move beyond description towards explanation of mind-brain relations.

Jeffrey $D$. Schall is in the Department of Psychology, Wilson Hall, Vanderbilt University, Nashville, Tennessee 37240, USA.

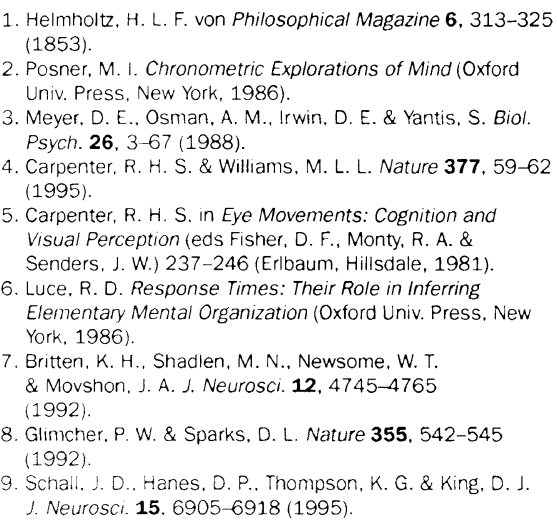

\section{Warm days, hot nights}

\section{D. Danny Harvey}

COMPUTER climate models driven by increasing carbon dioxide concentration predict that essentially the same warming should occur by day and by night. But this is not so. Between 1951 and 1990, records show that the average daily maximum of air temperature at the land surface has increased by only $0.28^{\circ} \mathrm{C}$, whereas the average increase in minimum daily temperature has been $0.84{ }^{\circ} \mathrm{C}$ - a factor of three larger. Why the discrepancy? Writing in Atmospheric Research ${ }^{1}$, Jim Hansen and colleagues Makiko Sato and Reto Ruedy conclude that the mean warming and lower diurnal temperature range can only be explained simultaneously by a combination of regional increases in aerosol optical depth and low-level cloudiness, and a large-scale warming factor such as greenhouse gas increases.

This conclusion is not altogether unexpected. Regions and seasons showing the strongest reduction of diurnal temperature range also show statistically significant increases in cloudiness. Cooling due to industrially produced sulphate aerosols either acting alone, or causing greater cloudiness - has been widely invoked to explain the discrepancy between model projections and observations, but so far only in qualitative arguments. Hansen et $a l$., in contrast, have used a three-dimen- sional atmospheric general circulation model to examine how the diurnal temperature cycle is affected by increases in carbon dioxide, cloudiness and sulphate aerosols, acting individually and together.

Sulphate aerosols are a by-product of sulphur oxide emissions, which in turn are produced industrially from the combustion of sulphur-containing fossil fuels. The primary radiative effect of sulphate aerosols is to increase the reflection to space of solar radiation, an effect which will weaken daytime heating but have little effect on night-time temperatures. But sulphate aerosols also serve as nuclei for cloud condensation, and, by increasing the number of small droplets, could indirectly increase cloud reflectivity and the lifetime of individual clouds, thereby increasing mean cloudiness. Cloudier skies should be particularly effective in reducing the diurnal temperature variation, as they reduce both solar heating by day and infrared cooling by night. Observations indicating that cloud albedos are indeed higher where sulphate concentrations are large ${ }^{2}$ and that increased cloudiness is associated with increasing sulphur emissions ${ }^{3}$ strengthen this connection.

Climate modellers distinguish between the 'equilibrium' response to a heating perturbation, which is the climate change that occurs once the system has fully adjusted to the imposed perturbation, and the transient or time-dependent response. Hansen and colleagues find that, in equilibrium, a $\mathrm{CO}_{2}$ increase capable of giving a mean warming of $0.5^{\circ} \mathrm{C}$ reduces the diurnal temperature amplitude by only $0.08^{\circ} \mathrm{C}$ averaged over all land. This is due in part to the direct increase in atmospheric infrared absorptivity caused by a higher $\mathrm{CO}_{2}$ concentration and in part to the increase in atmospheric water vapour content produced by the $\mathrm{CO}_{2}$-induced warming. The only realistic perturbation acting in isolation which can damp the diurnal cycle by the amount observed and change the mean temperature over land by $0.5^{\circ} \mathrm{C}$ is an increase in middle-level cloudiness over land by about 3 per cent - but this produces mean cooling rather than warming. To match both the mean warming and the damping of the daily cycle, Hansen and colleagues find that the known greenhouse gas heating must be combined with increases in aerosol optical depth and low-level cloudiness which reflect the regionally varying increase in estimated anthropogenic sulphate loading in the atmosphere.

In transient response experiments, however, the picture changes. Aerosol and cloudiness changes, and the direct effect of $\mathrm{CO}_{2}$ on atmospheric infrared absorptivity, quickly affect the diurnal cycle, whereas the change in mean temperature is delayed by two to three decades. This has two important consequences. First, $\mathrm{CO}_{2}$ and aerosol increases acting together can explain a much larger fraction - between one-third and a half - of the observed damping in diurnal temperature variation than implied by equilibrium simulations. A corollary is that a smaller increase in mean cloudiness over land, around $1 \pm 0.5$ per cent rather than 3 per cent, is required to explain the remaining damping. Second, and more important, as global mean temperatures come closer into balance with the greenhouse-gas heating perturbation (which must eventually happen), the difference between daytime and night-time warming can be expected to shrink. So, in the long run, daily maximum temperatures will increase by almost as much as the mean temperature. Nevertheless, to the extent that some effects of climate change depend on the rate of change of daily maximum temperature, a slower increase in daily temperature maxima could lessen the impact of global warming.

An important by-product of the effort to explain changes in the diurnal cycle is Hansen and colleagues' estimate that the combined cooling perturbation by aerosols and clouds is about half as large as the greenhouse-gas heating perturbation in the global mean. This result is independent of but consistent with attempts to calculate the relative aerosol and greenhouse-gas perturbations directly ${ }^{4}$. 